\title{
Commissioning and Alignment of the ATLAS Inner Detector using Cosmic Data
}

\author{
Daniel Kollar* \\ For the ATLAS Collaboration \\ CERN, Geneve \\ E-mail: daniel.kollarecern.ch
}

The ATLAS experiment is one of the two general purpose detectors at the LHC at CERN. ATLAS is equipped with a charged particle tracking system built on three sub-detectors, which provide high precision measurements made from a high detector granularity. The pixel and microstrip sub-detectors, which use the silicon technology, are complemented with the transition radiation tracker. The ATLAS detector is operational since 2008 and more than ten million cosmic tracks crossing the Inner Detector have been collected in 2008 and 2009. These data are used for the commissioning of the experiment. The alignment of the Inner Detector tracking system was performed using the 2008 cosmic data. The tracking performance obtained using this alignment is approaching the one obtained using Monte Carlo simulated with perfectly aligned geometry. The effect of systematic misalignments on physics measurements is being studied.

9th International Conference on Large Scale Applications and Radiation Hardness of Semiconductor Detectors

30 September - 2 October 2009

Florence, Italy

\footnotetext{
* Speaker.
} 


\section{The ATLAS Inner Detector}

The ATLAS experiment [1] is one of two general purpose experiments at the Large Hadron Collider (LHC) at CERN. It will record proton-proton collisions at a center-of-mass energy of up to $14 \mathrm{TeV}$. The ATLAS detector consists of four major subsystems: Inner Detector (ID), Electromagnetic Calorimeter, Hadronic Calorimeter and Muon Spectrometer.

The Inner Detector [2] is the main ATLAS tracking system occupying a cylindrical volume of $2.1 \mathrm{~m}$ in diameter and $6.2 \mathrm{~m}$ in length around the interaction point. It is enclosed inside the central super-conducting solenoid providing a $2 \mathrm{~T}$ axial field. The purpose of the ID is to precisely measure the trajectories of charged particles (tracks) created by proton-proton collisions inside the ATLAS detector. The ID tracking system covers a pseudo-rapidity range $|\eta|<2.5$.

The ID consists of two silicon subsystems: the Pixel Detector and the Semiconductor Tracker (SCT), complemented by the Transition Radiation Tracker (TRT) composed of straw tubes. Figure 1 shows a sketch of the ATLAS Inner Detector.

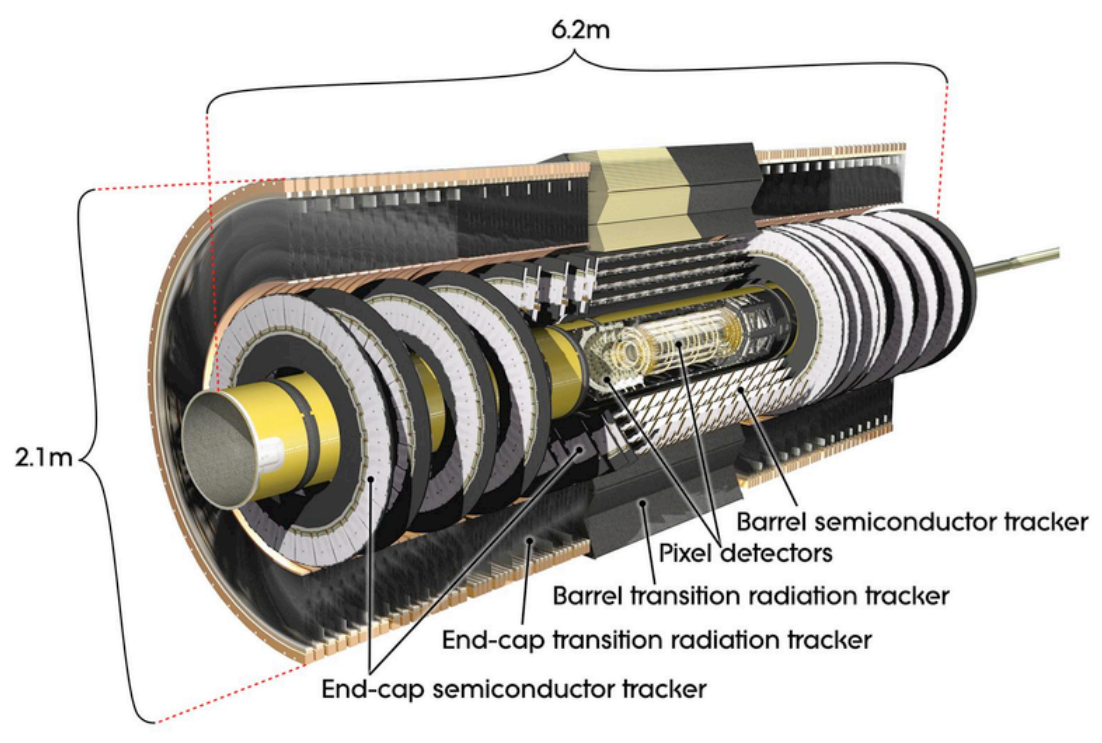

Figure 1: A sketch of the ATLAS Inner Derector.

The Pixel detector is the innermost sub-detector providing finest granularity. It consists of a barrel region with three cylindrical layers and two symmetric end-caps each containing three disks for tracking in the forward region. All pixel modules (1744 in total) are identical, with a sensor segmented in $50 \mu \mathrm{m} \times 400 \mu \mathrm{m}$ pixels providing a $2 \mathrm{D}$ readout with a resolution of $10 \mu \mathrm{m}$ and $115 \mu \mathrm{m}$ in the azimuthal $(R \phi)$ and transverse directions ( $z$ for the barrel and $R$ for the end-caps), respectively.

The SCT is made of four layers in the barrel region and nine disks in each of the two endcaps. Different types of modules have been installed in the SCT, all with the same components but differing in geometry. Each one of the 4088 modules is composed of two pairs of single-sided silicon micro-strip detectors glued back-to-back with a relative stereo angle of $40 \mathrm{mrad}$. The strip pitch is $80 \mu \mathrm{m}$ for the barrel and varying from $\sim 55 \mu \mathrm{m}$ to $\sim 90 \mu \mathrm{m}$ for end-cap modules due to 
their fan-out geometry. The intrinsic resolutions in the $R \phi$ and $z(R)$ directions are $17 \mu \mathrm{m}$ and 580 $\mu \mathrm{m}$, respectively.

The TRT is the outermost and largest of the ID sub-detectors. It is made of straw drift tubes which have a single hit resolution of $130 \mu \mathrm{m}$ in the $R \phi$ direction. The straw tubes are arranged in 32 modules in each of the three barrel layers and $2 \times 40$ end-cap wheels (176 modules in total). This setup provides an average of 30 TRT measurements per track.

\section{Cosmic data-taking}

From September to December 2008, ATLAS performed a series of global data-taking runs with Pixel, SCT and TRT fully integrated, recording 7.6 million cosmic ray tracks. Due to the geometrical acceptance for cosmic ray tracks, the number of tracks with hit information in SCT and Pixel detectors was reduced to 2 million and 420 thousand, respectively. The data was collected in configuration with and without the magnetic field.

This data was used for commissioning of the ATLAS ID including trigger, reconstruction, calibration and alignment, as well as for studying the tracking performance, and for gaining the operational experience in course of preparation for collisions. Presented here are the results of the alignment and tracking performance studies obtained using the cosmic ray data.

\section{Inner Detector alignment}

\subsection{The alignment problem}

The accuracy of the track reconstruction is limited by the precision with which the positions and orientations of individual modules are known. Neglecting the module deformations, such as bending and twisting, for each module 6 alignment degrees-of-freedom (DoF) can be defined: 3 translations and 3 rotations. This means that alone the silicon modules of the ATLAS ID have close to 35000 DoF. This poses a significant challenge for the alignment algorithms. The baseline goal is that the resolution of the track parameters should not be degraded by more than $20 \%$ by imperfect knowledge of the alignment of the ID. Ultimately the alignment should reach the precision of O(1 $\mu \mathrm{m})$ to allow for high precision Electroweak measurements, e.g. $W$ boson mass.

The initial precision of the ID module positions given by the construction accuracy is $\mathrm{O}(100$ $\mu \mathrm{m})$. For larger structures, i.e. barrel layers and endcap disks, the precision of installation is $100-$ $1000 \mu \mathrm{m}$. In order to achieve the required alignment precision track-based alignment algorithms are used. These are supplemented by the Frequency Scanning Interferometry (FSI) [3, 4] which is an optical alignment system installed for the SCT. In these proceedings only the track-based alignment is covered.

\subsection{The track-based alignment}

Several track-based alignment algorithms have been developed for the Inner Detector and are already implemented in the ATLAS software framework (Athena) [5]. All of them make use of track residuals, i.e., the distance from the reconstructed track position in the given detector module to the hit recorded in this module. The main idea is that the sum of the residuals over a large number 
of reconstructed tracks should be minimal for the aligned geometry. This can be formulated as a $\chi^{2}$ minimization problem with $\chi^{2}$ defined as

$$
\chi^{2}=\sum_{\text {tracks }} r^{T} V^{-1} r
$$

where $r(a, \pi)$ is a vector of track-hit residuals for a given track depending on both the alignment constants $a$ and the track parameters $\pi$, and $V$ is the covariance matrix of the hit measurements including the contribution from the multiple Coulomb scattering. The minimization is done with respect to the alignment parameters $a$ with the general solution for the alignment corrections $\delta a$ being

$$
\delta a=-\left(\sum_{\text {tracks }} \frac{d r^{T}}{d a} V^{-1} \frac{d r}{d a}\right)^{-1}\left(\sum_{\text {tracks }} \frac{d r^{T}}{d a} V^{-1} r\right) .
$$

Two different approaches can be followed when solving (3.2):

- Global $\chi^{2}$ alignment [6] . The solution given by Eq. (3.2) is calculated including all the correlations between the modules. The calculation is numerically very challenging as it involves solving a linear system with large number of parameters (close to 40000 for the full ID). The main two methods to do the calculation are the diagonalization and the fast-solving techniques. The Global $\chi^{2}$ approach is currently the baseline alignment algorithm used for the ATLAS Inner Detector.

- Local $\chi^{2}$ alignment $[7,8]$. The solution is calculated while neglecting the correlations between different detector modules, which reduces the alignment problem to solving a series of small linear systems. The inter-module correlations are restored by performing several iterations, updating the geometry of the detector and then re-fitting the tracks.

In addition to the $\chi^{2}$ minimization techniques, Robust alignment algorithm [9] has also been developed. It is based on shifting of modules according to their observed average residual offsets in an iterative way. In particular it takes advantage of the regions where modules overlap.

\subsection{Alignment strategy}

In the current implementation of the ATLAS Inner Detector software there is a separate code for silicon and TRT alignment. In the alignment procedure first the silicon detectors are fully aligned. Subsequently the Center-of-Gravity $(\mathrm{CoG})$ transform is applied which moves the CoG of the newly aligned silicon detector back to the origin of the ATLAS coordinate system. Afterwards the TRT is aligned starting from the aligned silicon detector. Finally the $\mathrm{CoG}$ is corrected again and the global ID alignment constants are obtained.

In order to address the realistic misalignments of the detector as well as to take into account the non-uniform cosmic-ray illumination of the $\mathrm{ID}^{1}$ the alignment is done on several different levels of granularity. First, the largest structures are aligned, for which largest misalignments are expected and at the same time smallest statistics is needed. Afterwards the degrees-of-freedom are added

\footnotetext{
${ }^{1}$ Cosmic-ray tracks are mostly vertical which leads to good illumination of the top and bottom parts of the barrel but poor track statistics for sides of the barrel and for the end-caps.
} 

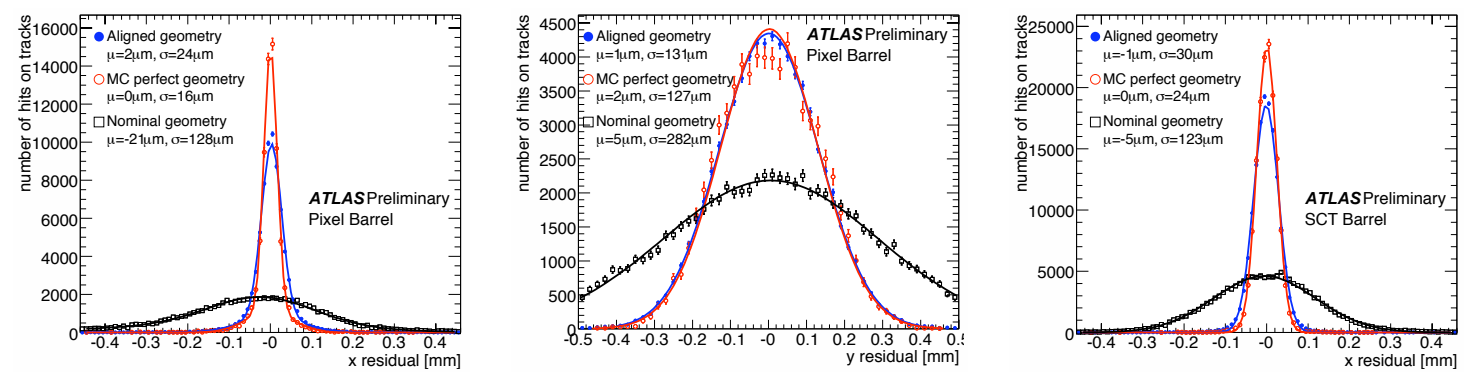

Figure 2: Pixel barrel residual distributions in $x$ (left) and $y$ (middle), and SCT barrel residual distribution in $x$ (right) before and after silicon alignment compared to the one obtained in simulation using perfect geometry.

depending on expected misalignments given by the construction. The alignment of the silicon detectors was done on four levels of granularity:

1) whole Pixel detector, SCT barrel, 2 SCT end-caps (24 DoF)

2) 6 Pixel barrel half-shells, 4 SCT barrel layers, 2 Pixel end-caps, 2 SCT end-caps ( 84 DoF)

3) 112 Pixel barrel staves, 176 SCT barrel staves, 2 Pixel end-caps, 2 SCT end-caps (1752 DoF)

4) individual Pixel and SCT barrel modules with only 2 most sensitive DoF per module: translation in $R \phi$ and rotation in the module plane (7136 DoF)

The alignment of the TRT barrel was performed in two levels of granularity:

1) TRT barrel wrt. silicon with 5 DoF excluding translation along the straw

2) internal alignment of TRT barrel modules with 5 DoF per module (480 DoF)

Currently a testing and validation of the new ATLAS track based alignment software is underway which aims to unify the usage of common algorithms and tools and simplify the overall alignment procedure. One of the gains due to the new structure is a possiblity to run the alignment of the entire Inner Detector at once making use of precise determination of the track momentum by the TRT in the silicon alignment. The plan is to use the new implementation from the start of the collision data-taking.

\section{Results}

\subsection{Residuals}

Alignment of the Inner Detector was performed using a mixture of data recorded with and without magnetic field in 2008 and a consistent set of alignment corrections was obtained. Figure 2 shows residual distributions for Pixel (left, middle) and SCT (right) before and after the alignment of the silicon using cosmic-ray data. Also shown are the distributions obtained from the Monte Carlo sample simulated with perfect geometry. Figure 3 shows residual distributions for TRT. A huge improvement in residual distributions after alignment can be seen for every sub-detector: the distributions are centered around zero and the widths approach those expected from simulation with perfectly aligned geometry. 


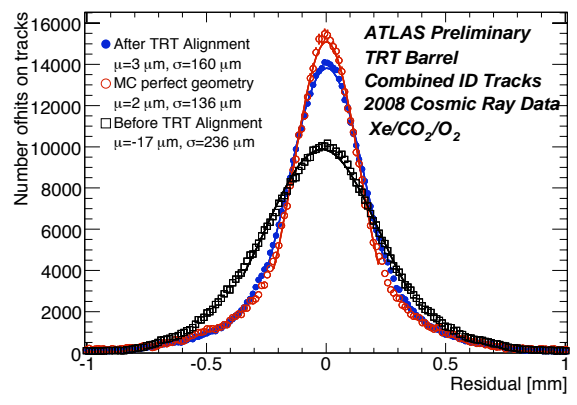

Figure 3: TRT barrel residual distribution before and after ID alignment compared to the one obtained in simulation using perfect geometry.

The cosmic ray data-taking has also continued during 2009. The residual distributions obtained in reconstruction of this data using the alignment constants obtained in 2008 are still centered at zero and only slightly broader, indicating stability of the detector over an extended period of time.

\subsection{Track-parameter resolutions}

While centering and width reduction of residual distributions is a necessary condition of a good alignment it is generally not sufficient. The quality of the alignment and its impact on physics measurement can be judged by studying the track-parameter resolutions. Such tracking performance studies can well be performed using cosmic tracks.

Since many cosmic-ray tracks cross both upper and lower hemisphere of the Inner Detector, by splitting the track into upper and lower half and refitting each half separately, one obtains two collision-like tracks. The resolution and bias of individual track parameters can then be directly measured by comparing the two tracks at the perigee. Figure 4 shows the difference in transverse impact parameter, $d_{0}$, and azimuthal angle, $\phi$, between the two tracks. Large offset and broad distribution can be seen for both parameters before alignment. After alignment huge improvement is observed with width of the distribution approaching the one obtained in MC simulation with perfectly aligned geometry.

An estimate of the track-parameter resolution can be obtained by dividing the width of the dis-
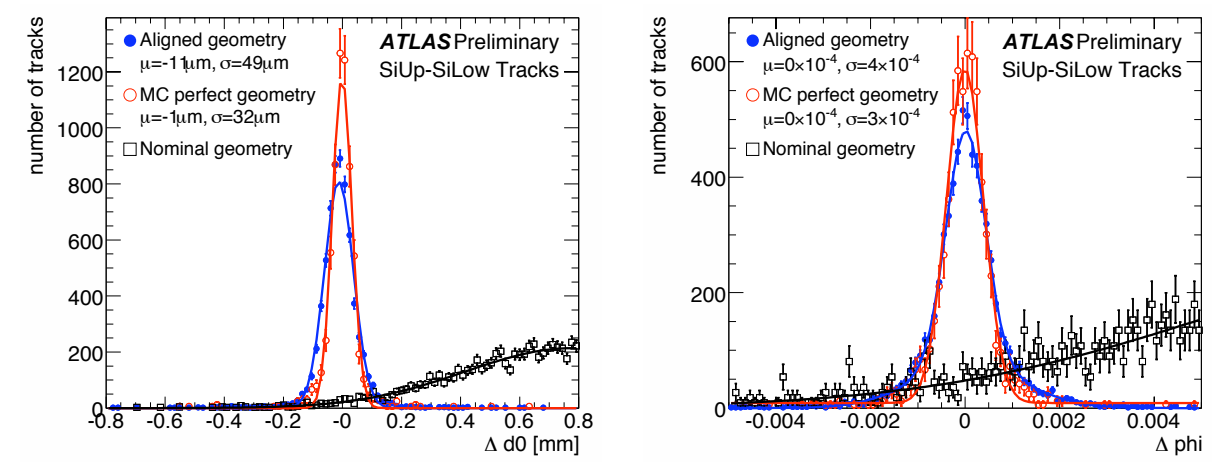

Figure 4: Difference in transverse impact parameter (left) and azimuthal angle (right) between two tracks obtained by splitting a single cosmic-ray track into upper and lower half and refitting each half separately. 

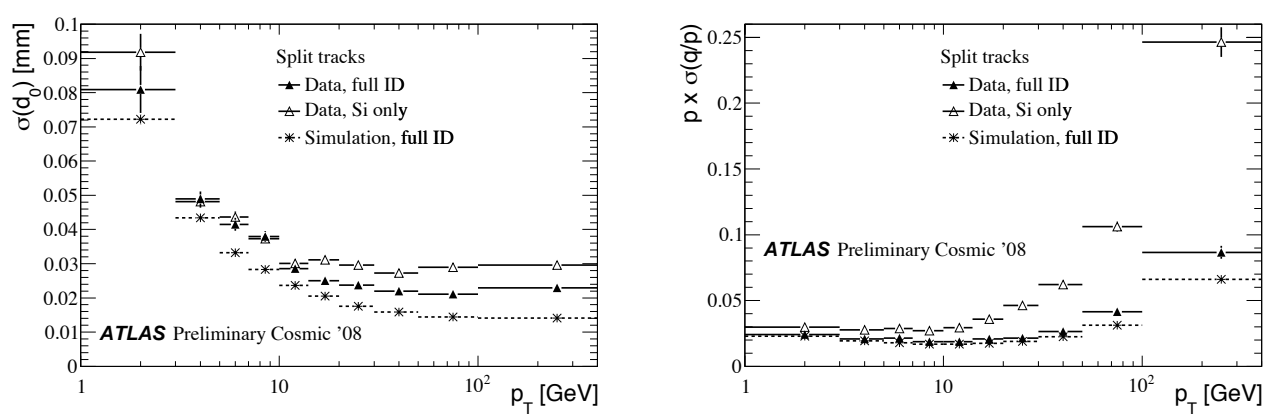

Figure 5: Transverse impact parameter (left) and relative momentum (right) resolution as a function of transverse momentum. Distributions for tracks reconstructed with full ID and silicon-only information are compared with the one obtained in MC simulation with perfectly aligned geometry.

tribution by $\sqrt{2}$. Figure 5 shows the resolution for $d_{0}$ and momentum, $p$, as a function of transverse momentum, $p_{T}$, for silicon-only tracks, full ID tracks, and tracks obtained in MC simulation with perfectly aligned geometry. In the low $p_{T}$ region, the worse $d_{0}$ resolution is due to multiple scattering effects. At higher values of $p_{T}$, the resolution is flat. Taking into account the TRT information improves the resolution.

The relative momentum resolution increases with higher $p_{T}$ due to stiffer tracks and a more difficult measurement of the sagitta. Including information from the TRT adds many more hits to the track and extends the lever arm, which helps improving the resolution especially for tracks with high $p_{T}$. For both shown track parameters the difference to the MC curve indicates the remaining misalignment.

\section{Weak modes}

Global distortions which preserve the helical trajectory of tracks and leave the $\chi^{2}$ unchanged while systematically biasing the track parameters are known as weak modes. These kind of distortions are difficult to be removed by the track based algorithms based on the minimization of the residuals while at the same time they represent a severe danger to physics results.

Several methods exist to remove (or at least, to keep under control as much as possible) the weak modes. These include for example the usage of different event topologies (cosmic ray tracks, beam halo, resonant mass constraint, etc.), redundant measurements within the same experiment (momentum measurement in the TRT and/or Muon Spectrometer), external constraints (survey measurements, FSI system, etc.), energy-momentum relation by including the information from the calorimeters, etc.

Impact of several different weak modes on physics measurements is being studied in ATLAS. An example is the so-called Curl deformation in which layers of barrel modules are shifted in $\phi$ by increasing (or decreasing) amount with increasing radius of the layer. Such deformation introduces a momentum bias: tracks of one charge are reconstructed with systematically larger momentum while the opposite charge tracks are reconstructed with systematically smaller momentum. The effect of Curl misalignment was studied using the reconstruction of the mass of the di-muon pair from the $Z \rightarrow \mu \mu$ decay. The size of the misalignment introduced before the reconstruction was 


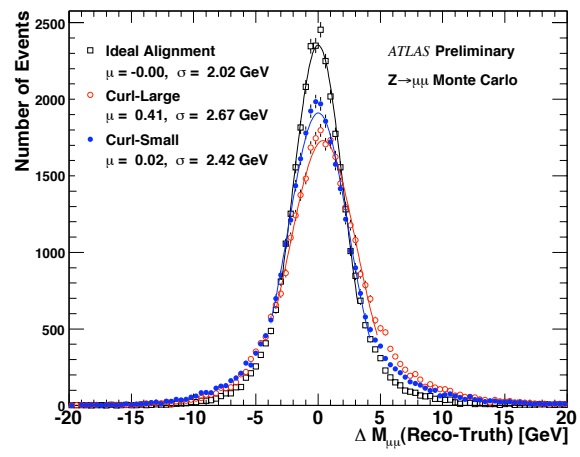

Figure 6: Resolution of the $Z$ mass for curl misalignment scenario before (CurlLarge) and after (Curl-Small) alignment compared to the perfectly aligned case.

such that the outermost silicon barrel layer was shifted by $300 \mu \mathrm{m}$ in $R \phi$. The $Z$ mass resolution shown in Fig. 6 labeled as Curl-Large demonstrates the effect of this deformation weak mode. The alignment procedure using only collision Monte Carlo was able to remove most of the bias caused by the Curl-Large resulting in a $Z$ mass resolution labeled as Curl-Small. In the case of curl, the resolution can further be improved by using the cosmic-ray tracks.

\section{Conclusions}

The ATLAS Inner Detector has been commissioned with 2008 and 2009 cosmic-ray data. The full statistics of the Fall 2008 cosmic-ray data-taking was analysed and used to align the ATLAS Inner Detector. Significant improvements in the track parameter resolutions were obtained. The analysis has substantially contributed to the understanding of the alignment of the ATLAS Inner Detector providing good starting conditions for the collision data-taking. Further improvement is expected after analyzing the 2009 cosmic-ray data.

Due to limitations of the data sample as well as those of the alignment procedure itself the ultimate accuracy of the alignment was not reached. Rapid improvements are expected with the first LHC collision data especially for the end-caps and for the weak mode removal.

ATLAS Inner Detector is well prepared for the first collisions.

\section{References}

[1] Aad G et al. (ATLAS) 2008 JINST 3 S08003

[2] ATLAS Collaboration (ATLAS) 1997 CERN-LHCC-97-16/17

[3] Coe P A, Howell D F and Nickerson R B 2004 Measur. Sci. Tech. 15 2175-2187

[4] Gibson S M et al. 2005 Optics and lasers in engineering 43 815-831

[5] ATLAS Computing TDR - 17, CERN-LHCC-2005-022

[6] Brückman de Renstrom P, Hicheur A and Haywood S 2005 ATL-INDET-PUB-2005-002

[7] Härtel R 2005 Diploma thesis, TU München, Germany

[8] Göttfert T 2006 Diploma thesis, Universität Würzburg, MPP-2006-118

[9] Heinemann F 2007 ATL-INDET-PUB-2007-011 (Preprint 0710 .1166) 